Copyright (C) 2005 IEEE. Reprinted from

IEEE/LEOS Workshop: Fibres and Optical Passive Components (2005 :

Palermo, Italy)

This material is posted here with permission of the IEEE. Such permission of the IEEE does not in any way imply IEEE endorsement of any of the University of Adelaide's products or services. Internal or personal use of this material is permitted. However, permission to reprint/republish this material for advertising or promotional purposes or for creating new collective works for resale or redistribution must be obtained from the IEEE by writing to pubs-permissions@ieee.org.

By choosing to view this document, you agree to all provisions of the copyright laws protecting it. 


\title{
Advances in Microstructured Fiber Technology
}

\author{
Optoelectronics Research Centre, \\ University of Southampton, \\ Southampton, SO17 1BJ, United Kingdom. \\ * Now at: The School of Chemistry and Physics, \\ University of Adelaide, \\ Adelaide, SA5005 Australia.
}

D.J. Richardson, F. Poletti, J.Y.Y. Leong, X. Feng, H. Ebendorff Heidepreim*, V. Finazzi, K.E. Frampton, S. Asimakis, R.C. Moore, J.C. Baggett, J.R. Hayes, M.N. Petrovich, M.L. Tse, R. Amezcua, J.H.V. Price, N.G.R. Broderick, P. Petropoulos and T.M. Monro*

\begin{abstract}
We review our recent progress in the area of microstructured fiber design, fabrication and applications with particular emphasis on the control of both the nonlinearity and dispersion.
\end{abstract}

\section{INTRODUCTION}

$\mathrm{T}$ The first work on microstructured optical fibers dates back to the early 1970's when a significant body of research was done on an effectively air-clad core supported on a thin structural membrane [1]. This work was directed at the development of low loss fibers for optical transmission and although impressive results were obtained, research into airclad fiber was soon stopped due to the successful development of vapour deposition based techniques for low loss preform fabrication.

Interest in the field of microstructured fibers was re-ignited in 1996 with the first demonstration of optical guidance within a complex 'holey' structure [2]. This new class of microstructured fiber, referred to as either Holey Fiber (HF), or Photonic Crystal Fiber (since the microstructure within the fiber is often highly periodic due to the fabrication processes used), provides a host of interesting and technologically enabling properties. Holey fibers possess a solid core surrounded by a cladding region that is defined by a fine array of air holes that extend along the full fiber length (see Fig.1). HFs are typically made of a single material, (usually pure silica), and guide light through a modified form of total internal reflection since the volume average refractive index in the core region of the fiber is greater than that of the surrounding regions. Note that the hole diameter (d) and pitch $(\Lambda=$ hole to hole spacing) which are the critical design parameters used to specify the structure of an HF are typically on the scale of the wavelength of light $\lambda$.

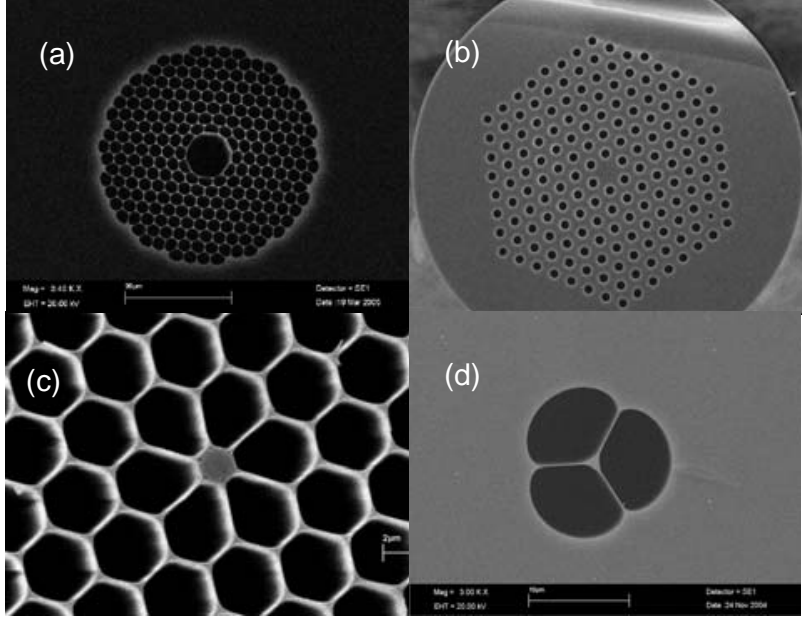

Fig.1: Some typical microstructured fiber types fabricated at ORC: (a) a PBG fiber for high optical power delivery; (b) a large mode area holey fiber ( $20 \mu \mathrm{m}$ core diameter); (c) a small core silica HF fabricated using capillary stacking which provides tight mode confinement and high optical nonlinearity; (d) a high nonlinearity HF in SF57 glass produced using an extrusion technique.

The fundamental physical difference between HFs and conventional fiber types arises from the way that the guided mode 'experiences' the cladding region. In a conventional fiber, this is largely independent of wavelength to first-order. However, in a holey fiber, the large index contrast between glass and air and the small structure dimensions combine to make the effective cladding index a strong function of wavelength. Short wavelengths remain tightly confined to the core, and so the effective cladding index is only slightly lower than the core index. However, at longer wavelengths, the mode samples more of the cladding, and so the effective index contrast is larger. This unusual wavelength dependence leads to a host of highly unusual and tailorable optical properties. One striking property is that fibers with a low air fill fraction 
$(d / \Lambda<0.4)$ can be single-moded regardless of the wavelength [3]. This property is particularly significant for broadband or short wavelength applications. Tailoring the scale of the cladding features allows the effective fundamental mode area of a holey fiber at $1.55 \mu \mathrm{m}$ to be varied over three orders of magnitude from $\sim 1 \mu \mathrm{m}^{2}$ to $1000 \mu \mathrm{m}^{2}$ [4]. Thus HFs can be seen to have a significantly broader range of optical properties than conventional optical fibers which, as well as being of fundamental scientific interest, opens up the possibility for new and technologically important fiber devices.

Although many HFs exhibit a periodic cladding structure this is not required in order to obtain guidance by average index effects [5]. However there is another class of microstructured fiber in which a periodic arrangement of air holes is essential to confine light. This class of fiber is referred to as photonic band gap fiber (PBGF) [6]. In PBGFs the periodic arrangement of holes in the cladding region of the fiber leads to the formation of a photonic band gap in the transverse plane of the fiber. Frequencies within this band gap cannot propagate in the cladding, and are thus confined to propagate within the core which acts as a defect in the otherwise 'perfect' periodic structure. It was in fact early failed attempts to produce PBGFs that resulted in the discovery of average index guiding Holey Fibers in 1996. It took several further years before microstructured fiber fabrication technology advanced to the point that PBGFs could be fabricated and this guidance mechanism demonstrated in practice [6]. PBG fibers offer a host of unique optical properties and represent an area of acute current interest.

Within this paper we shall review recent advances in the development of microstructured fiber technology with a primary emphasis on average index guiding structures. We shall review some of the latest advances in fabrication technology and touch briefly upon developments in the modeling and design of HF structures. Finally, we shall present recent results concerning the development of fibers for nonlinear applications which highlight some of the unique features of the technology, and discuss some of the applications that it has enabled.

\section{FIBER FABRICATION}

Holey fibers are typically fabricated by stacking an array of capillaries in a hexagonal configuration around a rod, which ultimately forms the core. The resulting preform is then drawn down to fiber dimensions using a conventional fiber drawing tower. If a large scale-reduction factor is required, a two-step drawing procedure is generally used. At this second stage, the microstructured region can be over-clad with a solid jacket, which allows extremely small structural dimensions to be achieved in a robust fashion. Dopants, e.g. germanium, aluminium, erbium, ytterbium, can also be incorporated into the individual stacked element to facilitate the development of fiber devices such as fiber lasers [8], amplifiers [9] and gratings [10].
By controlling the conditions under which the preform is drawn to fiber, the geometry of the fiber can be modified. For example, at high temperatures, the holes reduce in size due to surface tension effects. In this way a range of fiber profiles can be produced from one starting preform. Holey fiber technology has now reached the point that km-lengths of polymer-coated $\mathrm{HF}$ with losses as low as $0.28 \mathrm{~dB} / \mathrm{km}$ at $1.55 \mu \mathrm{m}$ [10] and tensile strengths comparable to those of conventional fiber are now possible. It is also important to note that it has been shown that advanced fiber drawing techniques such as fiber spinning previously developed to reduce the Polarisation Mode Dispersion (PMD) of conventional fibers are applicable to $\mathrm{HF}$ fibers without compromise to the fiber structure [11].

The fabrication of PBGFs is considerably more challenging than conventional fibers. However dramatic improvements have recently been obtained and low loss PBGFs can now be produced in practical lengths. PBGFs are also normally produced by the stack-and-draw technique. In this instance the air core is formed by removing a few elements from the centre of the stack. Various lattice structures and fiber core designs can be realized using this technique. For telecommunications wavelengths the hole pitch in the fiber are of the order a few $\mu \mathrm{m}$. A high air filling factor $(>0.9)$ is usually required to achieve a broad bandwidth and low-loss in silica PBGFs. In the most common design, air holes are arranged in a close-packed triangular lattice, and the core is composed of seven missing elements and is surrounded by 7-8 rings of holes (see Fig.1a). Fibers with bigger cores (19 elements) are also possible and offer the possibility of reducing the intensity of the mode at the glass air interface. This leads to benefits in terms of loss reduction although clearly working with a larger core can lead to issues in terms of the existence of higher order modes. PBG fiber losses as low as $1.7 \mathrm{~dB} / \mathrm{km}$ have been achieved in 19-hole core designs [12] and theoretical predictions show that losses of order $0.1 \mathrm{~dB} / \mathrm{km}$ might ultimately be obtained in this fiber type [13].

The emergence of microstructured fiber technology is particularly useful as it allows fibers to be made from just a single material, eliminating the need for two thermally, chemically and optically compatible glasses to form the fiber core and cladding as otherwise required within conventional fibers. HFs have now been made in a range of compound glasses [14-18] and polymers [19]. It is worth noting that the possibility of using new materials with lower melting points facilitates the use of techniques other than capillary stacking for preform production and indeed the first demonstrations of preform fabrication via techniques such as extrusion [20], builtin-casting [21] and drilling [22] have all now been reported. In particular, HF technology provides a simple and convenient route to realizing fibers in high nonlinearity glasses that might otherwise not be capable of being drawn into fiber form. To date, highly nonlinear soft glass HFs have been demonstrated for chalcogenide [14], lead silicate [15,16], bismuth silicate [18] and tellurite [17] glasses. We shall discuss the fabrication 

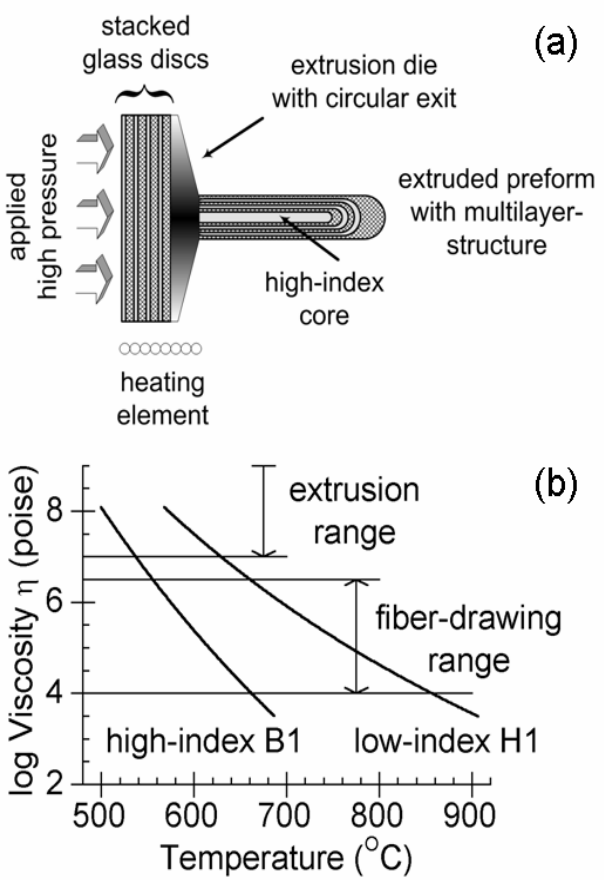

Fig.2: (a) All glass high-index core 1D MOF fabricated using a simple extrusion technique from alternating flat discs of two different glasses of suitably matched thermal and chemical characteristics (B1 and H1). (b) Plot showing the viscosity characteristics of the two glasses illustrating the temperature ranges for both preform fabrication and fiber drawing.

of an SF57 glass based HF exhibiting a record nonlinearity in detail in Section IV. It is also worth noting that many of these more exotic glass types, in particular the chalcogenides, offer extended transmission into the mid-IR. Microstructured fiber technology thus promises a new generation of fibers for mid-IR applications in medicine, sensing and defense.

Whilst HFs are typically pure air glass structures it is worth noting that MF concepts can also be applied to solid fibers made from two glasses with substantially different refractive indices, provided of course that these glasses are adequately matched in terms of their thermal and mechanical properties [23]. This opens up new fabrication possibilities and provides significant advantages for certain fiber types - not least in the sense that the fiber geometry is defined at the preform fabrication stage and that only the structure scale is defined at the draw stage.

In Fig.2a we illustrate a new extrusion technique for the production of a high-index-core, 1D, MOF based on two optical glasses with high index-contrast [24]. The microstructured preform with multiple coaxial rings is fabricated by extruding alternately stacked high- and low-index glass discs through a die of circular cross section.

The two glasses in this instance were a lead-oxide containing borosilicate glass $(\mathrm{PbO}>30 \mathrm{~mol} . \%)$ with a refractive index of


Fig.3: SEMs of two fibers of different ODs drawn from the same extruded preform showing preservation of the initial structure on two different dimension scales ((a) fiber $\mathrm{OD}=825 \mu \mathrm{m}$, (b) fiber $\mathrm{OD}=135 \mu \mathrm{m}$ ).

$\mathrm{n}=1.76$ at $1550 \mathrm{~nm}$ (referred as B1 hereafter), and a potassiumfluoride (KF) containing borosilicate glass with index $n=1.53$ at $1550 \mathrm{~nm}$ (referred as H1 hereafter). Fig.2(b) shows the viscosity curves of these two glasses measured using the parallel-disc technique. It can be seen that there is some thermal mismatch between these two glasses in the extrusion and fiber drawing regimes. However our results show that this thermal mismatch is within acceptable bounds. In our experiments $1.0 \mathrm{~mm}$ thick high-index glass discs were stacked alternately with $2.0 \mathrm{~mm}$ thick low-index glass discs. The stack of glass discs was heated to above the glass softening temperature (corresponding viscosity: $\sim 10^{9}-10^{7}$ poise). The stack was then extruded through the circular die exit under visco-elastic glass flow conditions to form a preform with multiple coaxial ring layers. The extruded preform was then reduced in scale on a fiber-drawing tower into cane with a $790 \pm 10 \mu \mathrm{m}$ outer-diameter (OD). An $80 \mathrm{~mm}$ long section of uniform cane was selected and inserted within a high-index B1 glass jacket-tube with $18 \mathrm{~mm}$ OD and $800 \pm 10$ $\mu \mathrm{m}$ inner-diameter (ID). Finally, this rod-in-tube assembly was pulled into a high-index-core 1D MOF. Fibers with a variety of ODs ranging from 825 to $115 \mu \mathrm{m}$ were produced.

Figs.3a and b show Scanning Electronic Microscope (SEM) photographs of this high-index-core 1D MOF with $825 \mu \mathrm{m}$ and $135 \mu \mathrm{m}$ OD, respectively. The high-index-core is surrounded by 9 alternate rings of low and high-index glass. Observe that the resulting core and rings are highly circular and concentric. By examining SEMs for fibers with ODs ranging from $825 \mu \mathrm{m}$ to $135 \mu \mathrm{m}$ we were able to demonstrate that the microstructure geometry of this high-index-core $1 \mathrm{D}$ MOF is dimensionindependent as expected and consistent with previous 
observations made in 2D-structured SOlid HOle (SOHO) fibers [23]. This is an important advantage of solid MOF relative to air-filled holey fiber since it allows the stable and repeatable definition of transverse structure that is essential for achieving optical fibers with pre-determined optical characteristics. Note that the diameter of the rings varies across the fiber due to the complex interplay of friction effects within the die during extrusion and the thermal mismatch between the two glasses. By adjusting the thickness of the starting discs to compensate this non-uniformity, it should be possible to achieve preforms with ring layers of controllable thickness (such as periodically spaced ring layers if required). Using this approach we have so far succeeded in making single mode fibers with losses below $4 \mathrm{~dB} / \mathrm{m}$. Further improvements in loss and fibers with specific dispersion characteristics are anticipated in the near future.

\section{FIBER MODELLING AND DESIGN TOOLS}

The simplest method for modelling the optical properties of HFs is the effective index model [4], which uses a (scalar) equivalent step-index fiber approximation. Although this model can provide some insight, it cannot accurately predict modal properties such as the dispersion and birefringence, which depend critically on the cladding configuration. Note that when the air fill fraction is large or the structure scale is small, it is necessary to use a full vector method. One general vector approach to describing the complex spatial index distribution in a HF involves decomposing the refractive index profile and modal fields into plane waves [25,26]. This technique is computationally intensive, since it does not take advantage of the localization of the guided modes in the fiber core. Multipole methods have also been developed to study HFs [27], and this method is well suited to evaluating the confinement losses and symmetry properties of single-material HF designs. A hybrid approach, which is efficient, since it uses localized functions to describe the guided modes, and accurate, because it uses plane waves to describe the index profile, is described in Refs [4,28]. Finally it is worth mentioning that FEM methods are also very well suited to modeling microstructured fibers and can be extremely efficient and fast.

Now that efficient techniques for modeling microstructured fibers have been developed there is a growing interest in developing inverse design techniques to design and optimize fiber structures with specific parameters. This is particularly important given the vast parameter spaces that are possible with this technology. For example, we have recently investigated the use of Genetic Algorithms to target fibers with specific combinations of desired properties [29]. Specifically, we used our GA approach to design dispersion flattened fibers for broadband nonlinear applications. We used the GA to minimize the integrated chromatic dispersion value over the wavelength range $1500 \mathrm{~nm}$ to $1600 \mathrm{~nm}$ for various generic holey fiber structures such as the one shown inset in Fig.4 (which is characterized by 6 structural parameters). We restricted the

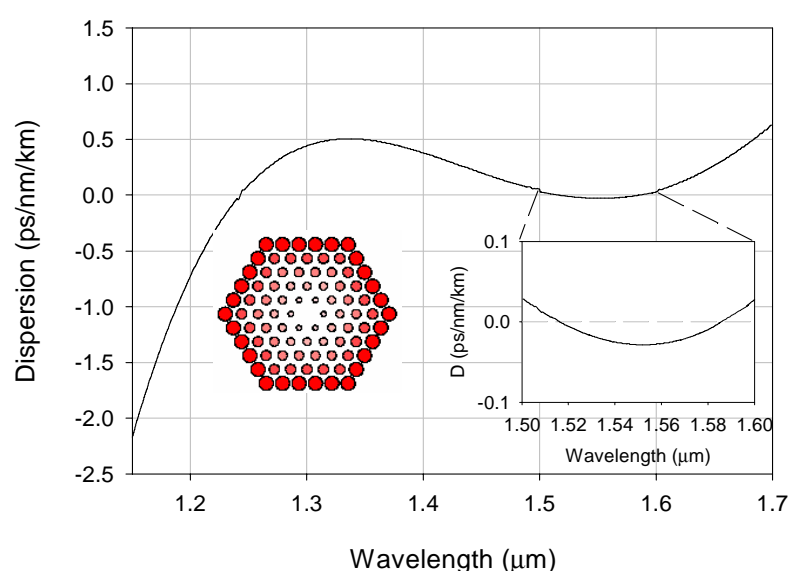

Fig.4: Calculated dispersion profile for the radially chirped pure-silica HF shown in the Inset, optimized using a GA. $\left(\Lambda=1.516 \mu \mathrm{m}, \mathrm{d}_{1}, \mathrm{~d}_{2} . . \mathrm{d}_{5}=0.317\right.$, $0.484,0.571,0.632,0.667 \mu \mathrm{m})$.

parameter space to cover only those designs offering relatively high effective nonlinearity. The results we obtained demonstrate that ultra-flat dispersion profiles can be achieved for this structure whilst maintaining a reasonably high effective nonlinearity $\left(\gamma=10.4 \mathrm{~W}^{-1} \mathrm{~km}^{-1}\right.$ for the case shown in Fig.4). Average dispersion slopes of less than $3 \cdot 10^{-3} \mathrm{ps} / \mathrm{nm}^{2} / \mathrm{km}$ were possible over the full target wavelength range. Our tolerance analysis showed that for this design a precision of less than $1 \%$ in hole size and position are necessary to control the dispersion characteristics with suitable accuracy. The GA approach represents a powerful design tool and can be used to optimize the design of many HF types. The area of inverse fiber design represents a key area of research and holds much promise for the development of a host of interesting new fibers.

\section{NONLINEAR FIBER TECHNOLOGY AND APPLICATIONS}

Arguably the most exciting prospect for microstructured fiber technology is the possibility of developing fibers with accurately controlled values of nonlinearity and dispersion. It is here that one can truly benefit from the large linear and nonlinear refractive index contrasts between glass and air, in order to realize fibers with truly unique and useful properties. By suitable control of the fiber transverse structure, and material choice, it is possible to envisage fiber designs with nonlinearities that are tailorable over more than 6-orders of magnitude. This opens up a host of new device possibilities ranging from nonlinear elements for telecommunications applications through to low nonlinearity fibers for high power laser delivery. In Table I we summarize a range of holey type structures and tabulate the ranges of both the nonlinearity per unit length $\gamma$ and loss that can be achieved currently, and indicate values that we expect to be realised in the future. The effective mode area $A_{\text {eff }}$ and $\gamma$ of a fiber at a wavelength $\lambda$ are related via $\gamma=2 \pi n_{2} /\left(\lambda A_{\text {eff }}\right)$ where $n_{2}$ is the nonlinear refractive index of the glass. 
In the low nonlinearity extreme we estimate that low loss PBG fibers with nonlinearities around 1000 times lower than existing SMF should be possible by minimizing the amount of light propagating within the solid regions of the fiber, and maximizing the amount of light within the low nonlinearity air core. (Air has 3 orders of magnitude less nonlinearity than glass which, in conjunction with the fact that fibers can be designed with $>99.5 \%$ of the mode in air, accounts for the massive reduction in effective nonlinearity). At the other extreme we can anticipate HFs with nonlinearities that are 10,000 times that of conventional SMF by making use of the tight mode confinement possible in small-core, high-NA HF, and the large material nonlinearities that can be achieved in high index materials such as chalcogenide glass. Note that once such high levels of nonlinearity can be achieved, e.g. to the point that devices of order $1 \mathrm{~m}$ become practical, then the relatively high losses of the high index base materials cease to be a major limiting issue for many important applications.

The high index contrast between air and glass allows for extreme values of waveguide dispersion and this can be used to control the large material dispersion associated with compound glasses at wavelengths of interest in the near IR - e.g. at $1550 \mathrm{~nm}$ for telecommunications, or around $1060 \mathrm{~nm}$ for incorporation within $\mathrm{Yb}$-doped fiber based devices. Such possibilities were previously exploited in silica HFs to obtain anomalous dispersion, and hence support of optical solitons [30] and supercontinuum generation [31] at pump wavelengths in the visible/near IR. Other work, such as that described in Section III above, has shown that waveguide dispersion can also be tailored to provide broadband dispersion-flattened properties [4,32]. To illustrate the potential for dispersion control in high nonlinearity fibers, and to indicate some of the compromises that are often required in terms of trading nonlinearity for dispersion control we describe some of our recent work on the fabrication of lead silicate glass HFs.

Lead silicate glass is proving a particularly promising material for high-nonlinearity HFs. In 2003 we reported a lead-silicate (Schott SF57 glass) HF with $\gamma=640 \mathrm{~W}^{-1} \mathrm{~km}^{-1}$ [33]. Here we show that by improving the fabrication process and optimizing the fiber design, values of $\gamma$ as high as $1860 \mathrm{~W}^{-1} \mathrm{~km}^{-1}$ at $1.55 \mu \mathrm{m}$ can be realized with improved fiber losses [34]. This $\gamma$ value approaches the ultimate limit for this material and represents the highest value of nonlinearity yet reported for an optical fiber. In addition, through a slight modification of our design, we show that we can tune the dispersion characteristics of the fiber to improve its performance for supercontinuum generation when pumped at wavelengths around $1.06 \mu \mathrm{m}$. This wavelength region is technologically significant in that it can be addressed using Yb-doped fiber lasers to realize all fiber SC sources.

Lead silicate glasses offer higher thermal and crystallization stability and less steep viscosity-temperature-curves than chalcogenide and heavy metal oxide glasses, although the material nonlinearity is lower. SF57 glass exhibits the highest

\begin{tabular}{|c|c|c|c|}
\hline \multicolumn{4}{|c|}{ TABLE I } \\
\hline Fiber Type & $\begin{array}{l}\gamma @ 1550 \mathrm{~nm} \\
(\mathrm{~W} \cdot \mathbf{k m})^{-1}\end{array}$ & $\begin{array}{l}\text { Loss / } \\
(\mathrm{dB} / \mathrm{m})\end{array}$ & $\begin{array}{c}\text { Typical } \\
\text { Applications }\end{array}$ \\
\hline $\begin{array}{l}\text { Optimised Silica } \\
\text { PBG fiber }\end{array}$ & $0.005 ?$ & $0.0001 ?$ & \multirow{7}{*}{$\begin{array}{l}\text { Laser Beam } \\
\text { Delivery, } \\
\text { High power pulse } \\
\text { compression. }\end{array}$} \\
\hline $\begin{array}{l}\text { PBG fiber } \\
\text { (Fig.1a) }\end{array}$ & 0.01 & 0.001 & \\
\hline $\begin{array}{l}\text { Large Mode } \\
\text { Area Fiber } \\
\text { (Fig.1b) }\end{array}$ & 0.1 & 0.001 & \\
\hline Standard SMF & 1 & 0.0002 & \\
\hline $\begin{array}{l}\text { Pure Silica small } \\
\text { core HF } \\
\text { (Fig.1c) }\end{array}$ & 70 & $\sim 0.04$ & \\
\hline $\begin{array}{l}\text { SF57 Small core } \\
\text { HF } \\
\text { (Fig.1d) }\end{array}$ & 1860 & $\begin{array}{l}\sim 2 \\
(0.3 \mathrm{~dB} / \mathrm{m} \\
\text { material })\end{array}$ & \\
\hline $\begin{array}{l}\text { Chalcogenide } \\
\text { Small core HF }\end{array}$ & $10,000 ?$ & $0.1 ?$ & \\
\hline
\end{tabular}

Table 1: Summary of nonlinear and loss characteristics of various current (bold) and possible future (italics) microstructured fiber types. Indicative application spaces for the various fiber types are also specified.

nonlinearity among commercially available lead silicate glasses. The high lead concentration of this glass leads to a high linear refractive index of 1.81 at $1550 \mathrm{~nm}$ with losses in the bulk glass as low as $0.3 \mathrm{~dB} / \mathrm{m}$ at $1550 \mathrm{~nm}$. The nonlinear index has been measured to be $4.1 \times 10^{-19} \mathrm{~m}^{2} / \mathrm{W}$ at $1060 \mathrm{~nm}$. Lead glasses also exhibit low softening temperatures of $\sim 500^{\circ} \mathrm{C}$, which allows the use of extrusion for fiber preform processing.

We used a three-step procedure for the production of our first fibers (Fig.5). A block of cylindrical shaped glass of outer diameter $(\mathrm{OD})=30 \mathrm{~mm}$ and height $=30 \mathrm{~mm}$ were first cut from a block of SF57 bulk glass using ultrasonic drilling and are used to extrude the structured preform and jacketing tube. Microstructured preforms, rod and tubes can be reproducibly fabricated in this way and good dimensional control can be achieved. The structured preform, which has an OD of $\sim 16 \mathrm{~mm}$, was annealed and then drawn on a fiber drawing tower into a smaller scale cane of about $1 \mathrm{~mm}$ OD. The jacketing tube is extruded with an OD of $\sim 10 \mathrm{~mm}$ and inner diameter (ID) of about $\sim 1 \mathrm{~mm}$ sufficient to allow the structured cane to be inserted within it. Finally, the annealed cane is inserted within the extruded jacketing tube and the assembly is drawn down to fiber. The feasibility of this approach for very small core high NA HF fabrication has been demonstrated in the ORC for a number of glasses. Careful adjustment of the tension applied during fiber drawing allowed us to accurately control the outer diameter of the fiber, and hence the dimensions of the inner core itself. HFs with core diameter in the range of 1.0-1.3 $\mu \mathrm{m}$ were produced from 2 different assemblies. From a single preform with a length of $\sim 15 \mathrm{~cm}$, we produced fiber lengths of $\sim 200 \mathrm{~m}$, wound on a spool in several bands of uniform outer 


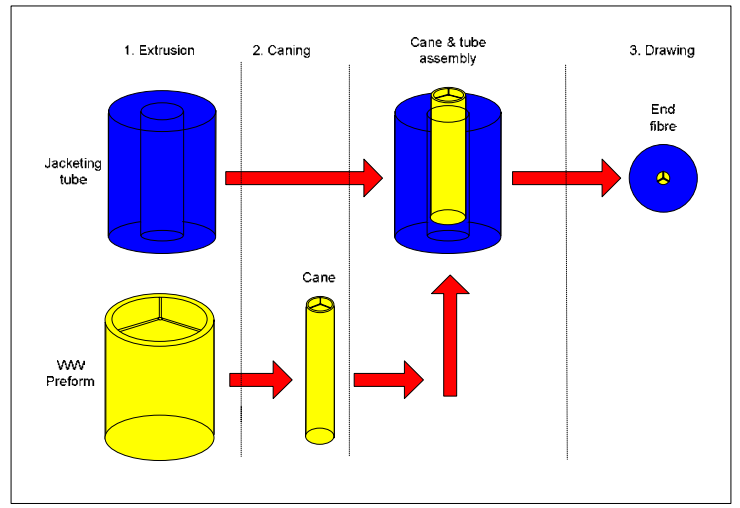

Fig.5: Fabrication scheme of the extruded lead silicate HF.

diameter in the range 100 to $150 \mu \mathrm{m}$. Note that the ratio between core size and fiber diameter can be changed via the choice of jacketing geometry and corresponding cane size, which allows the fiber diameter for a certain core size to be set.

We first performed a range of design calculations for a triangular core, SF57 HF structure (see Fig.6) in order to establish the variation in effective nonlinearity with core diameter of the HFs. The calculations were performed using FEMLAB, a commercial full-vector modal solver, based on the Finite Element Method. The results of these calculations are summarized in Fig.7 where we plot $\gamma$ versus core diameter for both operating wavelengths of $1550 \mathrm{~nm}$ and $1060 \mathrm{~nm}$. From this plot, we determined that a HF with a value of $\gamma$ approaching $2000 \mathrm{~W}^{-1} \mathrm{~km}^{-1}$ at $1550 \mathrm{~nm}$ is possible for core diameters in the range $0.6-1.0 \mu \mathrm{m}$. This fiber type is interesting for several nonlinear applications, offering the prospect of realizing compact nonlinear devices operating at low powers. Our smallest core fiber with a core diameter of $1 \mu \mathrm{m}$ (Fiber\#1) is close to the peak of the nonlinearity curve that corresponds to $1.55 \mu \mathrm{m}$ in Fig.7. Note that we targeted a diameter of $1 \mu \mathrm{m}$ as this provides a reasonable trade-off in terms of nonlinearity and the ease and efficiency of coupling into the fiber.

We first determined the guidance characteristics of Fiber\#1 at both $1 \mu \mathrm{m}$ and $1.55 \mu \mathrm{m}$, both experimentally by imaging the near-field of the guiding mode with an infrared camera and theoretically by calculating the mode profile from the SEM image. For both wavelengths, the predicted fundamental mode profile has a triangular shape in good agreement with the measured mode profile. The predicted effective mode areas are $0.84 \mu^{2}$ at $1.06 \mu \mathrm{m}$ and $1.1 \mu \mathrm{m}^{2}$ at $1.55 \mu \mathrm{m}$. A white light loss measurement was performed which showed a loss of $2.1 \mathrm{~dB} / \mathrm{m}$ at $1.06 \mu \mathrm{m}$ and $2.3 \mathrm{~dB} / \mathrm{m}$ at $1.55 \mu \mathrm{m}$. The reduced losses of this HF represent a significant improvement from our earlier demonstrations $(\sim 9 \mathrm{~dB} / \mathrm{m})$, which we attribute to advances in the fabrication through the use of ultrasonic cleaning of the preform. To explore the impact of the microstructure on the fiber properties, we also produced unstructured and unclad fibers (so-called bare fibers), which were drawn directly from

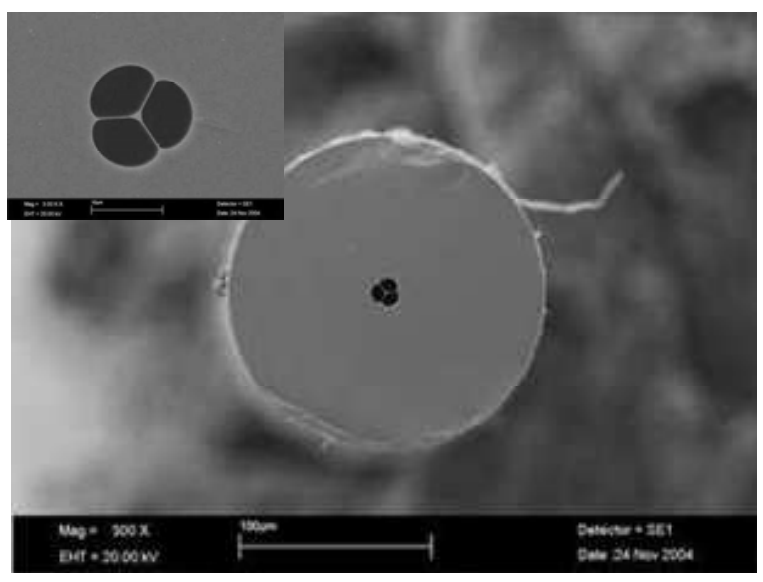

Fig.6: SEM of the SF57 glass HF Fiber\#1 (core diameter $\sim 1 \mu \mathrm{m}$ ).

extruded rods. For this heavily multimode solid SF57 fiber, we measured a loss of $1.0 \mathrm{~dB} / \mathrm{m}$ at $1 \mu \mathrm{m}$ indicating a modest increase in the background loss in going from bulk to fiber.

The effective nonlinear coefficient, $\gamma$, of the small-core HF at $1550 \mathrm{~nm}$ was estimated from the measurement of the nonlinear phase shift induced via self-phase modulation of a continuous wave, dual-frequency, optical beat signal propagated through the fiber. The results of this measurement are summarized in Fig. 9 and yield an estimate of $\gamma=1860 \mathrm{~W}^{-1} \mathrm{~km}^{-1}$, establishing this HF as the most nonlinear fiber ever produced. This number is slightly higher than our predicted value and we estimate it to be within $10-20 \%$ of the maximum value possible in this glass. In order to assess the dependence of the fiber dispersion characteristics on the core diameter of the HFs, the group velocity dispersion of a range of HFs of various core dimensions was calculated from the index profile of the fibers using FEM-based calculations. The zero-dispersion wavelength for this glass is $\sim 1.97 \mu \mathrm{m}$, however the strong waveguiding properties of the HF allow the large normal material dispersion at short wavelengths to be overcome. For a given wavelength, the dispersion value of small-core HFs increases as the core size decreases, due to enhanced waveguide dispersion. As a result, the zero-dispersion wavelength is shifted towards shorter wavelengths. The results of our calculations are summarized in Fig.8 where we plot the corresponding dispersion curves for different core diameters.

The plots show that a fiber with a zero-dispersion wavelength at $1060 \mathrm{~nm}$ (and also exhibiting a $\gamma=\sim 2000 \mathrm{~W}^{-1} \mathrm{~km}^{-1}$ at these wavelengths) is possible for a core diameter of $1.3 \mu \mathrm{m}$. This HF exhibiting an extremely small solid glass core and a very high air-filling fraction, not only displays unusual chromatic dispersion properties but also yields very high optical intensities per unit power. Thus it can be extremely well suited for nonlinear optic applications, where high effective nonlinearities, together with excellent control of chromatic dispersion, are essential for power-efficient devices. During the 


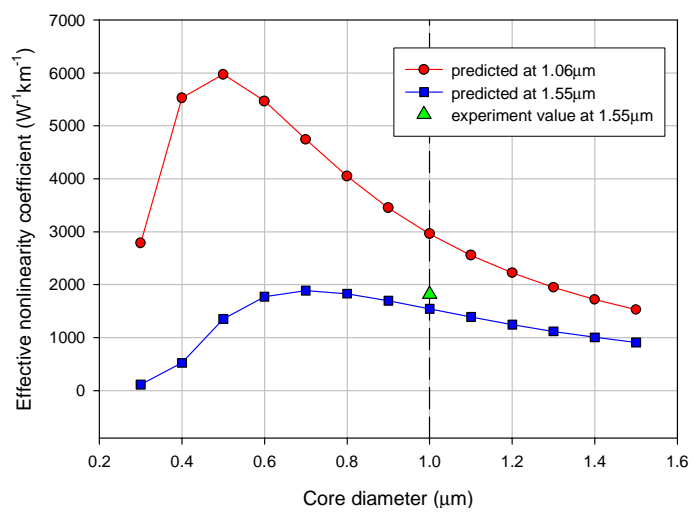

Fig 7: Effective nonlinear coefficient at $1.55 \mu \mathrm{m}$ and $1.06 \mu \mathrm{m}$.



Fig.8: Dispersion curves for bulk glass and HFs with different core diameters.

pull described in the previous section, we also fabricated a fiber with a slightly larger core $(1.3 \mu \mathrm{m})$, in accordance to these specifications (Fiber\#2). This fiber had similar properties in terms of mode shape and loss to Fiber\#1.

At present we have no ready way to measure the dispersion profile of short lengths of our fibers around $1 \mu \mathrm{m}$, so we chose to directly perform spectral broadening/supercontinuum experiments to highlight the fact that these HFs have a low dispersion in the $1 \mu \mathrm{m}$ range. We launched $200 \mathrm{fs}$ pulses at a repetition rate of $80 \mathrm{MHz}$ and a wavelength of $1.06 \mu \mathrm{m}$ with pulse energies up to $200 \mathrm{pJ}$ into short lengths of the two fibers. The pulses were first launched into $\sim 3 \mathrm{~m}$ of the $1.0 \mu \mathrm{m}$ core HF (Fiber\#1). Note, this length is far longer than required for the generation of supercontinuum and that so far we have made no attempt to optimize our set-up in terms of fiber length. For modest power levels (launched pulse energies below $\sim 45 \mathrm{pJ}$ ) we saw clear evidence of Raman soliton formation (Fig.10a). As we increased the power further, the spectral extent of the newly generated frequencies became broader (extended mainly towards the longer wavelength side) and the spectrum became smoother. We achieved a spectral broadening in excess of

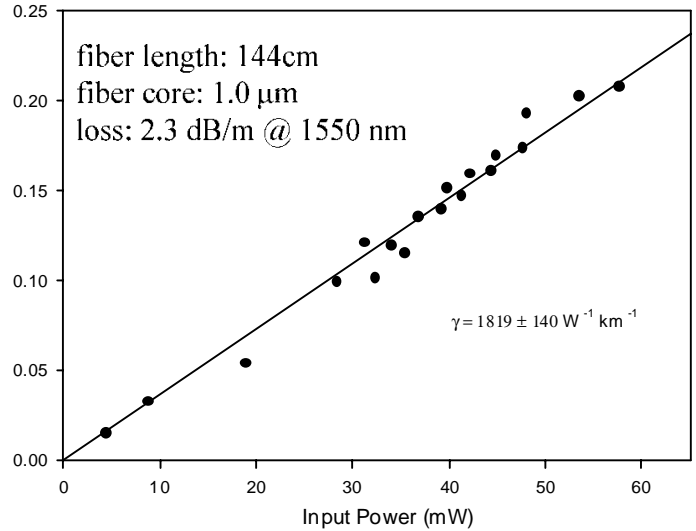

Fig. 9: Measured nonlinear phase shift as a function of input power yielding $\gamma=1860 \mathrm{~W}^{-1} \mathrm{~km}^{-1}$ from the slope of the linear fit for Fiber\#1 with 1 $\mu \mathrm{m}$ core.

$600 \mathrm{~nm}$ for launched pulse energies as low as $80 \mathrm{pJ}$. The spectral dip observed around $1.44 \mu \mathrm{m}$ is due to the $\mathrm{OH}^{-}$ absorption in the fiber which peaks at this wavelength.

We next experimented with a $\sim 50 \mathrm{~cm}$ long piece of the $1.3 \mu \mathrm{m}$ core HF. The zero-dispersion wavelength of this HF was much closer to the operating wavelength of the laser. This is most clearly evidenced by the significant spectral broadening due to SPM and four wave mixing at both longer and shorter wavelengths relative to the pump, observed at reduced pulse energy levels (see the $8 \mathrm{pJ}$ plot in Fig.10b). No such broadening is observed for fiber\#1 (see the $14 \mathrm{pJ}$ plot in Fig.10a). At higher pulse energies $(100-130 \mathrm{pJ})$ the spectral components in fiber\#2 spanned more than an octave and extended well into the shorter wavelength IR/visible regions of the spectrum.

These experiments show that lead silicate HFs hold promise for the development of compact nonlinear devices, such as all-fiber SC generators, operating at low powers. Application of the same fabrication approach to other more nonlinear glass materials than SF57 should ultimately allow for fibers with even higher values of effective nonlinearity per unit length.

It is also worth noting that as power levels from laser systems are increased, fibers with controllably low values of nonlinearity and good power handling characteristics are also finding increased uses in nonlinear applications. For example pure silica HFs with small holes and large hole spacing can be produced offering relatively large mode areas, well-defined low values of optical nonlinearity, and pure single mode guidance characteristics [35]. Fibers such as those shown in Fig.1b have thus found use in the nonlinear compression of pulses from high power fiber and thin disk lasers [36]. We show an example of such an application in Fig. 11 where a short length of LMA HF is used to generate SPM broadened from high power $800 \mathrm{fs}$ 

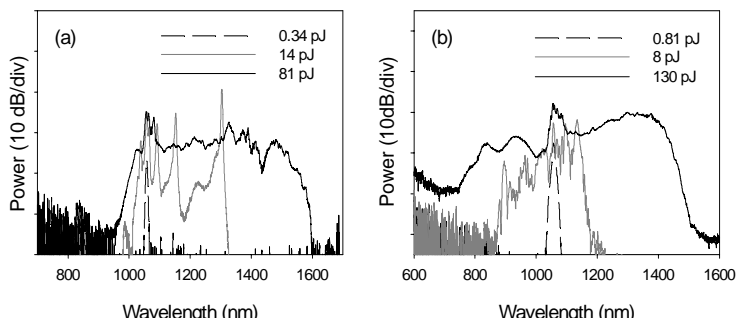

Fig.10: Spectra obtained at various launched pulse energies for (a) Fiber\#1, and (b) Fiber\#2.
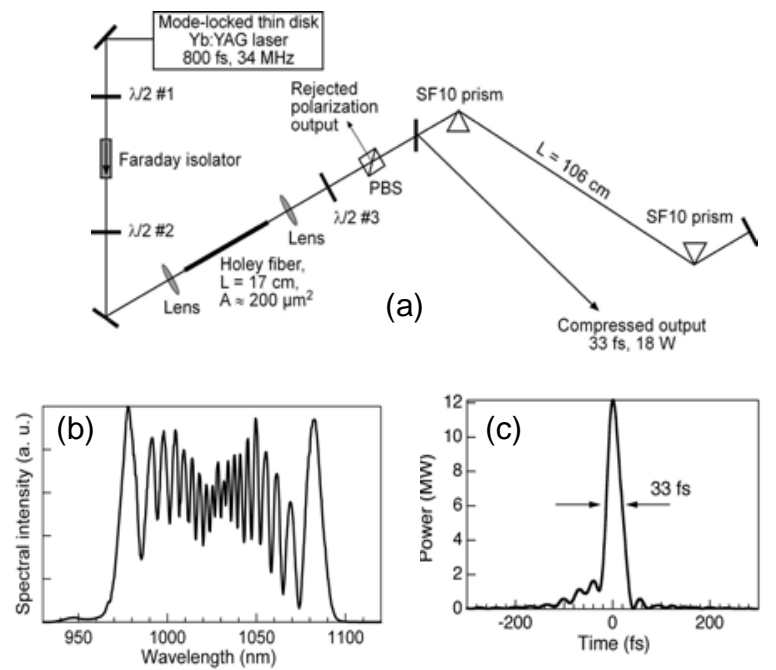

Fig.11: (a) Experimental configuration of the pulse compression scheme developed by our colleagues at ETH Zurich; (b) SPM broadened spectrum; (c) 33fs compressed pulses at the system output.

pulses generated within a mode-locked thin disk laser. The resulting pulses exhibit a largely linear chirp and are compressible to $\sim 33 \mathrm{fs}$ using a prism delay line. The peak power of the compressed pulses was $\sim 12 \mathrm{MW}$ and the average power through the system was $18 \mathrm{~W}$. This level of performance makes the source highly attractive for a host of ultrafast laser applications.

Although holey and conventional fibers can exhibit similar characteristics at any given wavelength, HFs have a distinct advantage for broadband and short wavelength applications due to their ability to be single-moded over a large wavelength range. The largest mode size that can be tolerated in practice is determined by macroscopic bending losses, and recent work demonstrates that HFs can possess at least comparable bend losses to similarly sized conventional fibers and offer potential advantages in terms of long term power handling capability [37].

\section{CONCLUSIONS}

In conclusion we have reviewed recent progress in the general area of microstructured fiber design fabrication with an emphasis on the design of fibers with controllable nonlinear properties. We consider the technology to hold great promise for use across a host of scientific and industrial sectors including the development of compact devices for all optical processing of signals within telecommunications through to the transmission of high power laser beams for industrial processing. We also highlight the versatility and flexibility of the technology in terms of the use of different materials. This will open up a range of new opportunities within new wavelength regimes, in particular within the mid-IR, for which it has been difficult to produce fibers using traditional approaches.

\section{ACKNOWLEDGMENTS}

J.Y.Y. Leong is supported by a Malaysian Government Studentship. S. Asimakis is supported by the Greek State Scholarships Foundation.

\section{REFERENCES}

[1] P. Kaiser, E.A.J. Marcatili, and S.E. Miller, “A new optical fiber,” Bell Sys. Tech. J. 52, 265-269 (1973).

[2] J. C. Knight, T. A. Birks, P. St. J. Russell and D. M. Atkin, "All-silica single-mode optical fiber with photonic crystal cladding," Opt. Letts. , 21, 1547-1549 (1996)

[3] T. A. Birks, J. C. Knight and P. St. J. Russell, "Endlessly single-mode photonic crystal fiber," Opt. Letts., 22, 961-963 (1997).

[4] T. M. Monro, D. J. Richardson, N. G. R. Broderick and P. J. Bennett, "Holey optical fibers: an efficient modal model," J. Lightwave Technol., 17, 1093-1102 (1999).

[5] T. M. Monro, P. J. Bennett, N. G. R. Broderick and D. J.Richardson, "Holey fibers with random cladding distributions," Opt. Letts., 25, 206208 (2000).

[6] J. C. Knight, J. Broeng, T. A. Birks, and P. St. J.Russell, "Photonic band gap guidance in optical fibers," Science, 282, 1476-1478 (1998).

[7] J Zhou, K Tajima,K. Nakajima, K. Kurokawa, C. Fukai, T. Matsui, I Sankawa, "Progress on low loss photonic crystal fibers", Opt. Fiber Tech., 11, 101-110, (2005).

[8] K. Furusawa, T. M. Monro, P. Petropoulos and D. J. Richardson, "Modelocked laser based on ytterbium doped holey fiber," Electron. Lett., 37, 560-561 (2001).

[9] J. H. V. Price, K. Furasawa, T. M. Monro, L. Lefort and D. J. Richardson, "A tuneable, femtosecond pulse source operating in the range 1.06 - 1.33 microns based on an Yb doped holey fiber amplifier", Postdeadline paper CPD1, Proc. CLEO'2001 (2001).

[10] B. J. Eggleton, P. S. Westbrook, R. S. Windeler,"Grating resonances in air-silica microstructured optical fibers," Opt. Letts., 24, 1460-1462 (1999).

[11] M.Fuochi, J.R.Hayes, K.Furusawa, W.Belardi, J.C.Baggett, T.M.Monro, D.J.Richardson, Polarization mode dispersion reduction in spun large mode area silica holey fibres, Optics Express, 12, 1972-7, (2004).

[12] B.J. Mangan, L. Farr, A. Langford, P.J. Roberts, D.P. Williams, F. Couny, M. Lawman, M. Mason, S. Coupland, R. Flea, H. Sabert, T.A. Birks, J.C. Knight and P. St.J. Russell "Low loss (1.7dB/km) hollow core photonic bandgap fibers", Proc. OFC 2004, PDP24, (2004).

[13] P.J. Roberts, F. Couny, H. Sabert, B.J. Mangan, D.P. Williams, L. Farr, M.W. Maso and A. Tomlinson, T.A. Birks, J.C. Knight and P. St.J. Russell "Ultimate low loss of hollow core photonic crystal fibers", Opt. Express, 13, 236-244, (2005).

[14] T. M. Monro, Y. D. West, D. W. Hewak, N. G. R. Broderick and D. J. Richardson, "Chalcogenide holey fibers," Electron. Lett., 36, 1998-2000 (2000).

[15] K.M.Kiang, K.Frampton, T.M.Monro, R.Moore, J.Tucknott, D.W.Hewak, D.J.Richardson, H.N.Rutt," Extruded single-mode nonsilica glass holey optical fibres", Electronics Letters, 38, .546-7, (2002). 
[16] V. Kumar, A. K. George, W. H. Reeves, J. C. Knight, P. S. Russell, F. G. Omenetto, and A. J. Taylor, "Extruded soft glass photonic crystal fiber for ultrabroad supercontinuum generation," Optics Express, 10, 15201525, (2002).

[17] Kumar, V.V.R., George, A.K., Knight, J.C., Russell, P.St.J.: 'Tellurite photonic crystal fibre', Opt. Express, 11, 2641-2645 (2003).

[18] H.Ebendorff-Heidepriem, P.Petropoulos, S.Asimakis, V.Finazzi, R.C.Moore, K.Frampton, F.Koizumo, D.J.Richardson, T.M.Monro, "Bismuth glass holey fibers with high nonlinearity", Optics Express, 12, 5082-7 (2004)

[19] M. A. van Eijkelenborg, M. C. J. Large, A. Argyros, J. Zagari, S. Manos, N. A. Issa, I. Bassett, S. Fleming, R. C. McPhedran, C. M. de Sterke and N. A. P. Nicorovici, "Microstructured polymer optical fiber", Opt. Express, 9, 319-327 (2001).

[20] K.M.Kiang, K.Frampton, T.M.Monro, R.Moore, J.Tucknott, D.W.Hewak, D.J.Richardson, H.N.Rutt, "Extruded single-mode nonsilica glass holey optical fibres", Electronics Letters, 38, 546-547 (2002).

[21] X.Feng, A.K.Mairaj, D.W.Hewak, T.M.Monro, "Towards high-indexglass based monomode holey fibre with large-mode-area", Electronics Letters, 40, 167-9, (2004)

[22] Mori, A., Shikano, K., Enbutsu, K., Oikawa, K., Naganuma, K., Kato, M., Aozasa, S. ' $1.5 \mu \mathrm{m}$ Band Zero-Dispersion Shifted Tellurite Photonic Crystal Fibre with a Nonlinear Coefficient of $675 \mathrm{~W}^{-1} \mathrm{~km}^{-1}$, ECOC 2004, 30th European Conference on Optical Communication, Th3.3.6 (2004).

[23] X.Feng, T.M.Monro, P.Petropoulos, V.Finazzi, D.Hewak," Solid microstructured optical fiber", Optics Express, 11, 2225-30, (2003).

[24] X.Feng, T.M.Monro, P.Petropoulos, V.Finazzi, D.J.Richardson, "Singlemode high-index-core one-dimensional microstructured fiber with high nonlinearity", Proc OFC 2005, (2005).

[25] E. Silvestre, M. V. Andres and P. Andres, "Biorthonormal-basis method for the vector description of optical-fiber modes," J. Lightwave Technol., 16, 923-928 (1998).

[26] J. Broeng, S.E. Barkou, T. Sondergaard and A. Bjarklev, "Analysis of air-guiding photonic bandgap fibers," Opt. Letts., 25, 96-98 (2000).

[27] T. P. White, R. C. McPhedran, C. M. de-Sterke, L. C. Botten and M. J. Steel, "Confinement losses in microstructured optical fibers," Opt. Letts., 26, 1660-1662 (2001).
[28] T. M. Monro, N. G. R. Broderick and D. J. Richardson, "Exploring the Optical Properties of Holey Fibers," in Nanoscale Linear and Nonlinear Optics, M. Bertolotti, Ed., New York: American Institute of Physics, (2000).

[29] F.Poletti, V.Finazzi, T.M.Monro, N.G.R.Broderick, V.Tse, D.J.Richardson, "Inverse design and fabrication tolerances of ultraflattened dispersion holey fibers", Optics Express, 13, 3728-3736 (2005).

[30] W. J. Wadsworth, J. C Knight, A. Ortigosa-Blanch, J. Arriaga, E. Silvestre and P. St. J. Russell,'Soliton effects in photonic crystal fibers at $850 \mathrm{~nm}$," Electron. Lett., 36, 53-55 (2000).

[31] J. K. Ranka, R. S. Windeler and A. J. Stentz, "Visible continuum generation in air-silica microstructure optical fibers with anomalous dispersion at $800 \mathrm{~nm}$," Opt. Letts., 25, 25-27 (2000).

[32] A. Ferrando, E. Silvestre, J. J. Miret, J. A. Monsoriu, M. V. Andres and P. St. J. Russell, "Designing a photonic crystal fiber with flattened chromatic dispersion," Electron. Lett., 35, 325-327 (1999).

[33] P.Petropoulos, H.Ebendorff-Heidepriem, V.Finazzi, RC.Moore, K.Frampton, D.J.Richardson, T.M.Monro," Highly nonlinear and anomalously dispersive lead silicate glass holey fibers", Optics Express 11, 3569-73 (2003).

[34] J.Y.Y.Leong, P.Petropoulos, S.Asimakis, H.Ebendorff-Heidepriem, R.C.Moore, K.Frampton, V.Finazzi, X.Feng, J.H.V.Price, T.M.Monro, D.J.Richardson, "A lead silicate holey with $\gamma=1860 \mathrm{~W}^{-1} \mathrm{~km}^{-1}$ at 1550 nm.”, OFC 2005 PDP22 (2005).

[35] J. C. Knight, T. A. Birks, R. F. Cregan, P. St. J. Russell and J. P. de Sandro, "Large mode area photonic crystal fiber," Electron. Letts., 34, 1346-1347 (1998).

[36] T. Sudmeyer, F. Brunner, E. Innerhofer, R. Paschotta, K. Furusawa, J. C. Baggett, T. M. Monro, D. J. Richardson, U. Keller, "Nonlinear pulse compression at high average powers levels by use of a large mode area holey fiber",Optics Letters, 28, 1951-53 (2003).

[37] J.C.Baggett, T.M.Monro, K.Furusawa, D.J.Richardson,”Understanding bending losses in holey optical fibers", Optics Communications 227, 317-335, (2003). 